\title{
Detailed images and distance measurements for eighteen dwarf irregular galaxies in the Canes Venatici cloud ${ }^{\star}$
}

\author{
L. Makarova ${ }^{1}$, I. Karachentsev ${ }^{1}$, L.O. Takalo ${ }^{1}$, P. Heinämäki ${ }^{2}$, and M. Valtonen ${ }^{2}$ \\ ${ }^{1}$ Special Astrophysical Observatory, N. Arkhyz, Stavropolsky kraj, 357147, Russia \\ 2 Tuorla Observatory, Turku, Finland
}

Received February 25; accepted July 21, 1997

\begin{abstract}
In the Canes Venatici cloud, eighteen dwarf irregular galaxies were observed in $B, V$ bands at the Nordic Optical Telescope under a seeing of one arcsec. Most of the galaxies having radial velocities $V_{0}<$ $500 \mathrm{~km} / \mathrm{s}$ have been resolved into stars for the first time. The galaxy distances were derived based on photometry of their brightest blue stars. Distances to some of the galaxies: UGC 6782, UGC 7131 and, probably, K 215, which are located at the CVn southern edge, were estimated to be about $15 \mathrm{Mpc}$, which is typical of the Virgo cluster outskirts. For two LSB galaxies, K 200 and K 215, the distances may be overestimated, probably because of a lack of young massive stars. For the remaining galaxies: UGC 7559, UGC 7599, UGC 7605, UGC 7639, UGC 7698, UGCA 290, UGCA 292, UGC 7866, UGC 8024, UGC 8638, UGC 8651, UGC 8760, and UGC 8833 the estimated distances range from 2.3 to $8.0 \mathrm{Mpc}$, indicating their actual membership in the CVn cloud.

Several objects studied here (UGC 7605, UGC 7639, UGC 8638, UGC 8833) have a well-resolved bluish core and regular yellow outer parts, which may point to composite (new and old) populations. The galaxy UGCA $292=$ CVn dwA has unusual global parameters: $(B-V)_{\mathrm{T}}=+0.08, M_{\mathrm{T}}=-11.4, M(\mathrm{HI}) / L_{B}=6 M_{\odot} / L_{\odot}$ and $M(\mathrm{HI}) / M_{\mathrm{T}}=0.7$, being, perhaps, one of the youngest known objects in the Local Universe.
\end{abstract}

Key words: galaxies: distances; irregular; stellar content

\section{Introduction}

A quarter of the 215 known galaxies with radial velocities $V_{0}<500 \mathrm{~km} / \mathrm{s}$ are located in a relatively small

Send offprint requests to: L. Makarova

* Table 2 to 19 are only available at the CDS via anonymous ftp 130.79.128.5 or via

http://cdsweb.u-strasbg.fr/Abstract.html area: R.A. $=\left(11.5^{\mathrm{h}}-14.0^{\mathrm{h}}\right), \mathrm{D} .=\left(+20^{\circ},+60^{\circ}\right)$ in the Canes Venatici constellation. This prominent concentration of the nearby galaxies (see Fig. 1) consists of several partially separated groups whose members were defined differently by several authors (Karachentsev 1966; de Vaucouleurs 1975; Kraan-Korteweg \& Tammann 1979; Vennik 1984; Tully 1988). The complex of galaxies in the Canes Venatici is populated mostly by objects of late morphological types. It does not have a common dynamical center usually associated with one luminosity-dominated galaxy.

Unlike other nearby groups of galaxies concentrated around M 81, M 101 and NGC 5128, the diffuse cloud in the Canes Venatici has not many galaxy distances known. Until 1995, the distance moduli determined from Cepheids and the brightest stars were obtained only for four galaxies: IC 4182 (Sandage \& Tammann 1982), DDO 154 (Carignan \& Beaulieu 1989), DDO 168 (Bresolin et al. 1993) and UGC 8508 (Karachentsev et al. 1994). Recent distance estimates based on the brightest stars have been derived for 11 irregular dwarf members of the cloud by Georgiev et al. (1997) and Makarova et al. (1997). Below, we present new photometric distances for 18 galaxies in the area under consideration. The rest of the galaxies in the Canes Venatici complex have been observed at the $6 \mathrm{~m}$ telescope of Special Astrophysical Observatory of the Russian Academy of Sciences and the $2.56 \mathrm{~m}$ Nordic Optical Telescope. The reduction of the data obtained is now near completion.

\section{Observations, data reduction and photometric results}

The observations of 18 dwarf irregular galaxies in Canes Venatici were carried out in 1995 February using a TEC $1024 \times 1024$ chip at the prime focus of the $2.56 \mathrm{~m}$ Nordic Optical Telescope (NOT) at La Palma. The CCD size was $24.58 \times 24.58 \mathrm{~mm}$, which yielded a total field of $3 \times 3$ arcmin. We observed the galaxies under good seeing conditions in Johnson $B$ and $V$ bands. The observational log is presented in Table 1. 


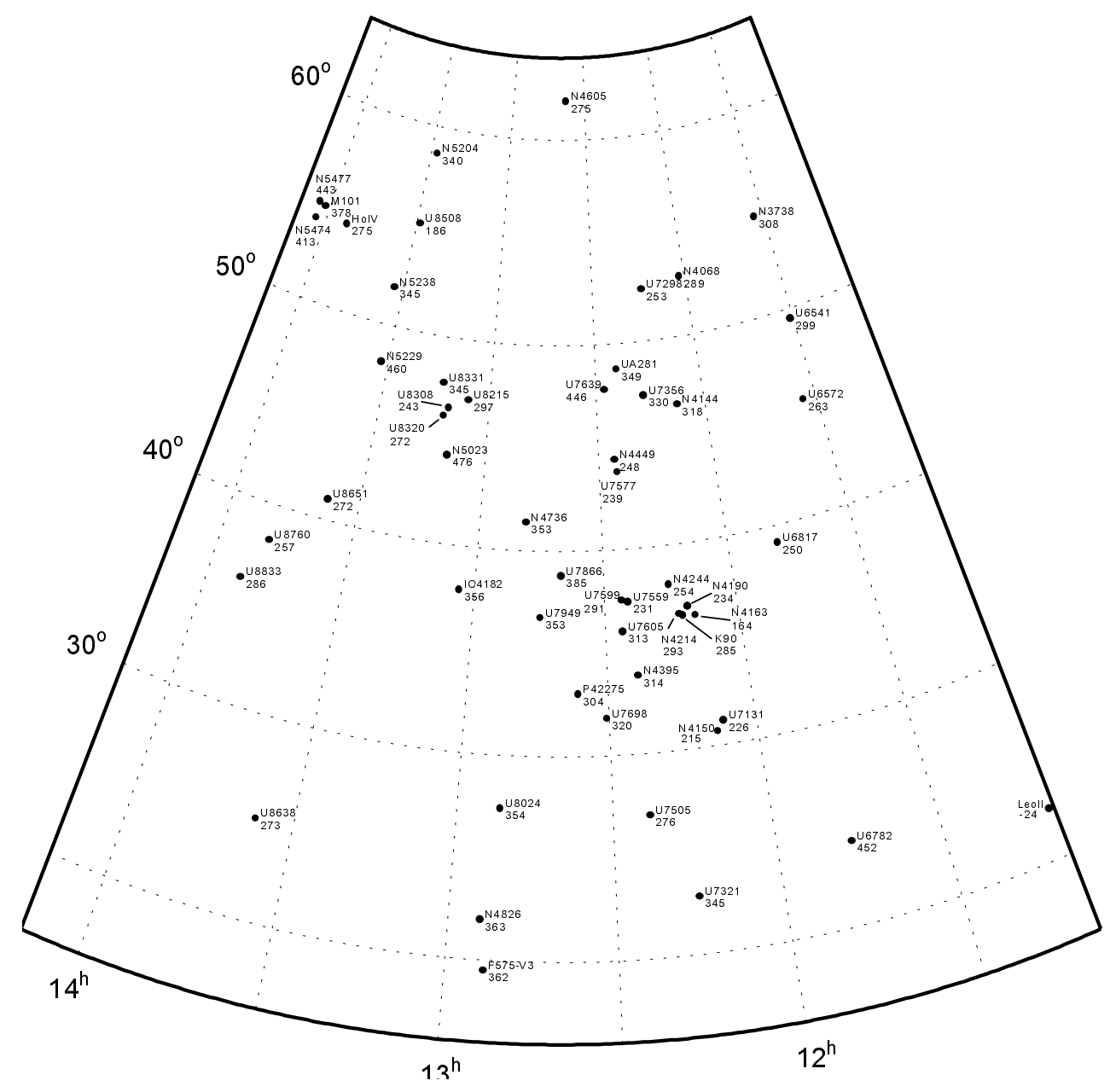

Fig. 1. The distribution of galaxies with corrected radial velocities $<500 \mathrm{~km} / \mathrm{s}$ in the Canes Venatici constellation in equatorial coordinates. Each galaxy is marked by its NGC or UGC number and its radial velocity (in $\mathrm{km} / \mathrm{s}$ )

The initial processing of the data was performed with IRAF (CCDRED package) using bias, dark and twilight flatfield frames obtained during the observing nights. Cosmic events filtering was done with CCDPHOT package.

The CCD frames of the 18 observed dwarf galaxies are presented either in $B$ or in $V$ band in Figs. 2-19. Most of the galaxies have been resolved into stars for the first time.

The photometry of stellar objects was carried out using MIDAS implementation of the point spread function (PSF) photometry programs DAOPHOT and ALLSTAR (Stetson 1987). The PSF was obtained for each frame from bright, well isolated stars, then PSF fitting of all stellar objects found in the frame was performed simultaneously by ALLSTAR. Object lists for each galaxy were combined according to positional identity (with a deviation of no more than 1.5 pixels) of the star images in different bands. The standard photometric error in $B, V$ and SHARP parameters of ALLSTAR were taken into account during the star selection process. Mostly objects with $-2.5 \leq$ SHARP $\leq 2.5$ and a standard photometric error $\leq 0.2$ were kept.

Our ALLSTAR magnitudes are based on the first estimate of stellar magnitudes with small aperture $(4-8$ pixels) to exclude contamination by neighbouring stars. Therefore, an aperture correction was made. We obtained this correction using PSF stars by plotting growth curves for each image. All objects found, except PSF stars, were subtracted from the images before this process. The aperture correction was then calculated as the difference between the asymptotic magnitude and the magnitude within a small aperture from the first estimate in ALLSTAR. We estimate mean errors of this correction as: $0.03^{\mathrm{m}}$ in $B, 0.03^{\mathrm{m}}$ in $V$ and $0.06^{\mathrm{m}}$ in $I$ band.

For the calibration, the photometric standards from Landolt's (1992) list were observed during the run. An aperture photometry of standard stars was done to obtain their magnitudes. After correction for atmospheric 
Table 1. Observational log

\begin{tabular}{|c|c|c|c|c|c|}
\hline Name & Date & Filter & $\begin{array}{c}\text { Exposure } \\
\text { (sec) }\end{array}$ & $\begin{array}{c}\text { Seeing } \\
\left({ }^{\prime \prime}\right)\end{array}$ & Airmass \\
\hline \multirow[t]{2}{*}{ UGC 6782} & 7.02 .95 & $B$ & 500 & 0.9 & 1.04 \\
\hline & & $V$ & 500 & 0.9 & 1.04 \\
\hline \multirow[t]{2}{*}{ UGC 7131} & 5.02 .95 & $V$ & 600 & 1.8 & 1.09 \\
\hline & & $I$ & 600 & 1.7 & 1.09 \\
\hline \multirow[t]{2}{*}{ UGC 7356} & 7.02 .95 & $V$ & 300 & 0.8 & 1.08 \\
\hline & & $B$ & 500 & 0.9 & 1.08 \\
\hline \multirow[t]{2}{*}{ UGC 7559} & 4.02 .95 & $V$ & 600 & 0.9 & 1.03 \\
\hline & & $B$ & 600 & 0.9 & 1.02 \\
\hline \multirow[t]{2}{*}{ UGC 7599} & 4.02 .95 & $V$ & 600 & 0.7 & 1.01 \\
\hline & & $B$ & 600 & 0.7 & 1.01 \\
\hline \multirow[t]{2}{*}{ UGC 7605} & 6.02 .95 & $V$ & 300 & 1.0 & 1.03 \\
\hline & & $B$ & 600 & 1.1 & 1.02 \\
\hline \multirow[t]{2}{*}{ UGC 7639} & 7.02 .95 & $V$ & 300 & 0.8 & 1.08 \\
\hline & & $B$ & 300 & 0.9 & 1.08 \\
\hline \multirow[t]{2}{*}{ UGC 7698} & 6.02 .95 & $V$ & 600 & 1.3 & 1.08 \\
\hline & & $B$ & 600 & 1.2 & 1.08 \\
\hline \multirow[t]{2}{*}{ UGCA 290} & 6.02 .95 & $V$ & 300 & 1.1 & 1.10 \\
\hline & & $B$ & 300 & 1.3 & 1.10 \\
\hline \multirow[t]{2}{*}{ UGCA 292} & 5.02 .95 & $V$ & 600 & 1.3 & 1.08 \\
\hline & & $B$ & 600 & 1.4 & 1.08 \\
\hline \multirow[t]{2}{*}{ UGC 7866} & 6.02 .95 & $B$ & 600 & 1.2 & 1.02 \\
\hline & & $V$ & 300 & 1.2 & 1.02 \\
\hline \multirow[t]{2}{*}{ UGC 7949} & 6.02 .95 & $B$ & 600 & 1.1 & 1.03 \\
\hline & & $V$ & 300 & 1.1 & 1.02 \\
\hline \multirow[t]{2}{*}{ UGC 8024} & 5.02 .95 & $B$ & 300 & 1.2 & 1.10 \\
\hline & & $V$ & 300 & 1.2 & 1.10 \\
\hline \multirow[t]{2}{*}{ K 215} & 6.02 .95 & $V$ & 600 & 1.0 & 1.05 \\
\hline & & $B$ & 600 & 1.1 & 1.05 \\
\hline \multirow[t]{2}{*}{ UGC 8638} & 6.02 .95 & $B$ & 600 & 1.2 & 1.03 \\
\hline & & $V$ & 600 & 1.0 & 1.02 \\
\hline \multirow[t]{2}{*}{ UGC 8651} & 5.02 .95 & $V$ & 600 & 1.4 & 1.04 \\
\hline & & $B$ & 600 & 1.5 & 1.03 \\
\hline \multirow[t]{2}{*}{ UGC 8760} & 5.02 .95 & $V$ & 600 & 1.2 & 1.01 \\
\hline & & $B$ & 600 & 1.4 & 1.01 \\
\hline \multirow[t]{2}{*}{ UGC 8833} & 5.02 .95 & $V$ & 600 & 1.0 & 1.02 \\
\hline & & $B$ & 600 & 1.4 & 1.02 \\
\hline
\end{tabular}

extinction, the following equations for transformation into Johnson-Cousins standard system were derived:

$V-v=0.023(b-v)+24.98$

$B-V=1.034(b-v)-0.09$

$V-I=1.117(v-i)+0.83$,

where the capital letters are Johnson-Cousins magnitudes and the small letters are instrumental ones. We estimate the following zero-point errors of this transformation: $0.04^{\mathrm{m}}$ in $V, 0.03^{\mathrm{m}}$ in $B-V$ and $0.05^{\mathrm{m}}$ in $V-I$. Adding quadratically these errors to those of the aperture correction, we obtain the resulting zero-point errors: $0.05^{\mathrm{m}}$ in $V, 0.05^{\mathrm{m}}$ in $B-V$ and $0.08^{\mathrm{m}}$ in $V-I$.

The results of the stellar photometry are presented in Tables 2-19 which can also be accessed in computerreadable form in the CDS. All the tables are organized as follow: (1) the ordinal number of the star; (2), (3) $X, Y$ coordinates of the star in pixels; $(4)$ - the apparent magnitude in $V$ band; $(5)$ - the $(B-V)$ colour index; $(6),(7)$ - the formal DAOPHOT error in $V$ and $(B-V)$ respectively; (8) - the mean of $B$ and $V$ SHARP value.

To determine the total magnitude of a galaxy, concentric circular apertures were used. The total magnitude in each band was defined to be the magnitude at which the curve of growth flattened. Background stars and distant galaxies were clipped before. We estimate the resulting error as $0.05^{\mathrm{m}}$ in $B$ and $0.05^{\mathrm{m}}$ in $V$.

\section{Distance estimates}

Colour-magnitude diagrams (CMDs) were constructed for our photometric data to reveal the brightest blue $(B-V<$ $0.4)$ and red $(B-V>1.6)$ stars. All of CMDs show a prominent blue bump with only a few or absent red stars. The photometric limit of our data (about $24^{\mathrm{m}}$ in $V)$ as well as the actually low number of red supergiants in dwarf irregulars can be the reasons for this situation. Therefore, the very few cases of distance estimation via the brightest red stars are presented just for reference. Our CMDs should not be too heavily contaminated by background stars because of the high galactic latitude of the objects in the Canes Venatici cloud.

Following Sandage \& Tamman (1974a-d) and de Vaucouleurs $(1978 \mathrm{a}, \mathrm{b})$ we measured the galaxy distance moduli using the mean magnitude of three brightest blue stars, $\langle B(3 B)>$. Taking into account its dependence on the total magnitude of the parent galaxy, $B_{\mathrm{T}}$, we have:

$$
\mu_{0}(B)=1.51 \times<B(3 B)>-0.51 \times B_{\mathrm{T}}-A_{B}+4.14
$$

where $A_{B}$ is the galactic extinction. The coefficients were calibrated using the galaxy distances derived from cepheids (Karachentsev \& Tikhonov 1994).

The results of our measurement and some basic parameters of the dwarf galaxies under consideration are presented in Table 20. We used the PGC-ROM catalog (Paturel et al. 1992) as a source of global parameters of the galaxies. We also obtained the total $B$ magnitude and the colour index from our frames, where it was possible. These data are marked with "c" in Table 20. The Table contains the following columns: (1) - the galaxy name; (2) -the standard diameter in arcmin; $(3)$ - the total $B$ magnitude; $(4)$ - the total colour index $(B-V)$ measured within the standard diameter; $(5)$ - the value of the galactic extinction; (6) -the heliocentric radial velocity $(\mathrm{km} / \mathrm{s})$; (7) - the radial velocity with respect to the centroid of the Local Group according to Karachentsev \& Makarov (1996); (8), (9) -the mean magnitude and the mean colour for the three brightest blue stars in the galaxy; (10) - the distance modulus of the galaxy; (11) - the corresponding distance in Mpc. 
Table 20. Basic parameters and distance estimations for the dwarf irregular galaxies in the Canes Venatici

\begin{tabular}{|l|l|l|c|c|c|c|c|c|c|c|}
\hline Name & $a_{25}$ & $B_{\mathrm{T}}$ & $(B-V)_{\mathrm{T}}$ & $A_{B}$ & $V_{\mathrm{h}}$ & $V_{0}$ & $\langle B(3 B)>$ & $<B-V>_{3}$ & $\mu_{0}(B)$ & $D$ \\
\hline UGC 6782 & $1.6 \mathrm{c}$ & $15.39 \mathrm{c}$ & $0.67 \mathrm{c}$ & 0.04 & +524 & +452 & 22.81 & -0.02 & 30.69 & 13.7 \\
UGC 7131 & 1.5 & $15.8 \mathrm{c}$ & $0.7: \mathrm{c}$ & 0.05 & +253 & +226 & $>23$ & - & $>30.8$ & $>14$ \\
UGC 7356 & 0.9 & $15.75 \mathrm{c}$ & $0.75 \mathrm{c}$ & 0.00 & +272 & - & - & - & - & - \\
UGC 7559 & 3.2 & $14.12 \mathrm{c}$ & $0.32 \mathrm{c}$ & 0.00 & +218 & +231 & 20.92 & +0.07 & 28.53 & 5.1 \\
UGC 7599 & 2.0 & $14.98 \mathrm{c}$ & $0.32 \mathrm{c}$ & 0.00 & +278 & +291 & 21.65 & -0.01 & 29.19 & 6.9 \\
UGC 7605 & 1.1 & $14.76 \mathrm{c}$ & $0.36 \mathrm{c}$ & 0.02 & +308 & +313 & 20.96 & -0.16 & 28.24 & 4.4 \\
UGC 7639 & 2.3 & $14.13 \mathrm{c}$ & $0.58 \mathrm{c}$ & 0.00 & +382 & +446 & 21.58 & -0.09 & 29.52 & 8.0 \\
UGC 7698 & $5.0 \mathrm{c}$ & $13.15 \mathrm{c}$ & $0.35 \mathrm{c}$ & 0.03 & +333 & +320 & 20.88 & +0.08 & 28.93 & 6.1 \\
UGCA 290 & 0.7 & $15.74 \mathrm{c}$ & $0.26 \mathrm{c}$ & 0.00 & - & - & 20.59 & +0.06 & 27.20 & 2.8 \\
UGCA 292 & 1.0 & $16.10 \mathrm{c}$ & $0.08 \mathrm{c}$ & 0.02 & +308 & +304 & 20.88 & -0.07 & 27.44 & 3.1 \\
UGC 7866 & 3.4 & $13.75 \mathrm{c}$ & $0.38 \mathrm{c}$ & 0.00 & +359 & +385 & 20.03 & +0.06 & 27.37 & 3.0 \\
UGC 7949 & 2.0 & $15.12 \mathrm{c}$ & $0.32 \mathrm{c}$ & 0.04 & +333 & +353 & 22.32 & +0.02 & $30.09:$ & $10.4:$ \\
UGC 8024 & $2.6 \mathrm{c}$ & $14.17 \mathrm{c}$ & $0.27 \mathrm{c}$ & 0.02 & +376 & +354 & 20.72 & -0.05 & 28.18 & 4.3 \\
K 215 & 1.2 & $16.52 \mathrm{c}$ & $0.22 \mathrm{c}$ & 0.03 & +419 & +362 & 23.65 & +0.01 & $31.40:$ & $19.1:$ \\
UGC 8638 & 1.2 & $14.44 \mathrm{c}$ & $0.44 \mathrm{c}$ & 0.00 & +274 & +273 & 19.86 & +0.05 & $26.77:$ & $2.3:$ \\
UGC 8651 & 2.4 & $14.45 \mathrm{c}$ & $0.34 \mathrm{c}$ & 0.00 & +203 & +272 & 20.46 & -0.04 & 27.66 & 3.4 \\
UGC 8760 & 2.2 & $14.45 \mathrm{c}$ & $0.29 \mathrm{c}$ & 0.00 & +193 & +257 & 21.04 & -0.12 & 28.54 & 5.1 \\
UGC 8833 & 0.9 & $15.15 \mathrm{c}$ & $0.32 \mathrm{c}$ & 0.00 & +228 & +286 & 20.61 & +0.06 & 27.53 & 3.2 \\
\hline
\end{tabular}

Below we note some properties of the galaxies observed.

UGC 6782 = DDO 97. This irregular galaxy from van den Bergh's (1966) list is hardly resolved into stars. Within its apparent boundary we can distinguish about a dozen faint bluish stars. Using the mean magnitude $<B(3 B)>=22.81^{\mathrm{m}}$ of the three brightest blue stars (Nos. 13, 9 and 15) and $B_{\mathrm{T}}=15.39^{\mathrm{m}}$ we derive a distance modulus $\mu_{0}=30.69$, which is typical of the Virgo cluster galaxies. Probably, the galaxy actually belongs to the Virgo periphery, and its low radial velocity is caused by the considerable peculiar motion with respect to the Virgo center.

UGC 7131. Unlike other galaxies in our sample, this one was observed in the $V$ and $I$ - bands only. It is entirely unresolved into stars. The Table 3 data refer to foreground stars and, probably, distant compact galaxies. The apparent absence of blue stars brighter than $23.0^{\mathrm{m}}$ yields the galaxy distance: $D>14 \mathrm{Mpc}$. As in the previous case, UGC 7131 may also belong to the Virgo cluster periphery. Note that a very blue star $\mathrm{N}^{\circ} 10$ with $V-I=-0.80$ is projected onto the galaxy northern side.

UGC 7356. The galaxy is unresolved on our frames. Taking into account its regular shape and yellow colour, we may consider UGC 7356 as a distant dwarf system of $\mathrm{dE} / \mathrm{dSph}$ type. Its radial velocity was measured by Thuan \& Seiter (1979). However, UGC 7356 lies only 5 arcmin away from the southern spiral arm of NGC 4258, which has a radial velocity $V_{\mathrm{h}}=+450 \mathrm{~km} / \mathrm{s}$ and a $\mathrm{HI}$ line width of about $420 \mathrm{~km} / \mathrm{s}$. That is why the radial velocity estimate for UGC 7356 should be considered as fictitious, because of confusion with the HI flux of the neighbouring spiral.
UGC $7559=$ DDO 126. This irregular dwarf system has been resolved into stars by Hopp \& Shulte-Ladbeck (1995) and Georgiev et al. (1997). On our CCD frames we detected and measured 192 stars. The colour-magnitude diagram for them is presented in Fig. 20. From the three brightest blue stars with $\langle B(3 B)\rangle=20.92$ we obtained the distance modulus $\mu_{0}=28.53$. Note that some rather red stars are present in the main body of the galaxy. If the brightest one (No. 57) is assumed to be a red supergiant, then using the relation

$$
\mu_{0}(R 1)=1.10 \times V(R 1)-0.10 \times B_{\mathrm{T}}-0.76 \times A_{B}+7.00
$$

we derive the "red" distance modulus $\mu_{0}(R 1)=28.80$, in agreement with the "blue" one. Expression (2) reflects a slight correlation between the luminosity of the brightest red supergiant and of its parent galaxy (Karachentsev et al. 1994). According to Hopp \& Shulte-Ladbeck (1995) and Georgiev et al. (1997), in UGC 7559 the brightest blue stars are slightly brighter $\left(20.77^{\mathrm{m}}\right.$ and $20.58^{\mathrm{m}}$, respectively) giving a smaller distance modulus: $28.42^{\mathrm{m}}$ and $27.97^{\mathrm{m}}$, respectively.

UGC $7599=$ DDO 127. The galaxies DDO 126 and DDO 127 are separated by 15 arcmin, and their radial velocities differ by $60 \mathrm{~km} / \mathrm{s}$ only. Nevertheless, their spatial distances turn out to be different. Unlike its neighbour, DDO 127 has a regular shape with a considerable brightness gradient towards the center. We found the distance modulus of UGC 7599 to be equal to 29.19 from the brightest blue stars (see CMD for the galaxy in Fig. 21). However, the distance estimate via the brightest red star (No. 160) yields a modulus of 29.84, which differs largely from the blue one. 
UGC 7605. The faint outlying part of the galaxy has a more regular shape than its patchy core. According to our aperture photometry the integrated colour of the galaxy increases smoothly from $B-V=+0.19$ in the center to +0.38 within the faintest isophotes. This colour gradient indicates clearly the existence of a young blue stellar population in the center of the galaxy as well as an older population dominated at the edge. The galaxy distance estimate via the brightest blue stars $\left(\mu_{0}=28.24\right)$ is in agreement with the red one $\left(\mu_{0}=28.05\right)$.

UGC 7639. Similarly to the previous object this galaxy shows a regular outlying shape. The galaxy integrated colour grows smoothly from $B-V=+0.30$ to +0.60 , suggesting the presence of different stellar populations. Based on the luminosity of the brightest blue stars (Nos. 12, 17, 27), UGC 7639 is situated at a distance of $8.0 \mathrm{Mpc}$. The bright blue star No. 33 in the outer part of the galaxy is probably a foreground object.

UGC $7698=$ DDO 133. This is a low surface brightness galaxy with a standard angular diameter of 5.0 arcmin. Because of its large size, the outer parts of the galaxy are left from our CCD frame. The distance modulus from the three brightest blue stars $\left(\mu_{0}(3 B)=28.93\right)$ does not correspond to the distance modulus from the brightest red star $\left(\mu_{0}(1 R)=26.97\right)$. Since other blue and red stars may occur outside the CCD frame, our distance estimate should be considered uncertain.

UGCA $290=$ Arp $211=$ VV 42. This patchy dwarf galaxy looks like an interacting binary system (Vorontsov-Velyaminov 1959). In the "Atlas of peculiar galaxies" (Arp 1966) it looks well resolved into stars. Recently we observed this galaxy with the 6-m telescope in $B, V$ bands and derived a distance modulus of $27.58 \mathrm{mag}$ from the brightest blue stars. Our present estimate (from stars Nos. $5,11,13) \mu_{0}(3 B)=27.20$ differs slightly from the previous one. We did not consider the stars No. 12 and 14 in the distance estimation due to their location in the extremely crowded region which may lead to overestimation of the star brightness.

UGCA $292=$ PGC $42275=$ CVn dwA. The very low surface brightness and the presence of some blue stellar complexes characterize the galaxy structure. According to our photometry, the system has an extremely blue total colour: $B-V=0.08$. Probably, this is the case of a very young galaxy undergoing active star formation process. The VLA observations of Lo et al. (1993) revealed a larger size for the galaxy in neutral hydrogen than in the optical light. The HI mass-to-light ratio for UGCA 292 is 6.1 in solar units, which is one of the highest value among the known galaxies. With a distance of $3.1 \mathrm{Mpc}$ derived by us, the galaxy HI mass-to-total mass ratio reaches about $70 \%$. The brightest blue object No. 23 lies at the edge of the galaxy may be a foreground star.

UGC $7866=$ IC $3687=$ DDO 141. Extending over the entire CCD field of view, the galaxy image shows many blue stars and associations as well as a few red stars.
Our estimate of UGC 7866 distance based on the three brightest blue stars is 27.37. However, the red modulus, $\mu_{0}(1 R)=28.43$, differs significantly from the blue one.

UGC $7949=$ DDO $147=K 200$. The galaxy has a low surface brightness without prominent clumps. All stars except for one in the galaxy are fainter than $V=22^{\mathrm{m}}$ which leads to a distance of $10.4 \mathrm{Mpc}$. However, if the galaxy is a moderatly young system without massive blue stars, its distance may be overestimated. Thus this estimate is an upper limit.

UGC $8024=$ DDO 154. The galaxy has a very asymmetric shape. Its standard optical diameter is 2.6 arcmin. According to Hoffman et al. (1993), in HI, the galaxy extends to 26 arcmin, i.e. 10 times the optical diameter. Photometry of the stellar population of the galaxy was made by Carignan \& Beaulieu (1989) and Hopp \& Shulte-Ladbeck (1995). The former authors estimated the galaxy distance to be $\leq 4 \mathrm{Mpc}$, via the brightest blue stellar objects. It became clear from a comparison of their data with ours that, the authors treated some multiple stars as single ones. According to our photometry of the brightest blue stars the galaxy distance is $4.3 \mathrm{Mpc}$.

$\mathbf{K} \mathbf{2 1 5}=\mathbf{F 5 7 5}-\mathbf{v 3}$. This very low-surface-brightness galaxy was discovered first by Karachentseva (1968), and then studied by Schombert et al. (1992). Within its apparent boundary we detected only a dozen faint bluish stars. Based on the three brightest stars we obtained a galaxy distance $D=19 \mathrm{Mpc}$ which suggests that the galaxy may be a member of the Virgo cluster. However, the galaxy distance may be overestimated because of the lack of young massive stars at the present stage of the galaxy evolution.

UGC $8638=$ VV 133. Some bright blue stellar complexes are clearly identified against the regular amorphous body of the galaxy. The integrated colour of the galaxy increases smoothly from +0.27 to +0.48 outwards. Except for the blue object No. 7 (which is a contact binary on the V-frame) the three brightest blue stars (Nos. 18, 20, 23) lie too much above the other stars (see CMD in Fig. 22). Perhaps, they are compact stellar clusters rather than stars. In this case, the derived distance of $2.3 \mathrm{Mpc}$, should be considered as a lower limit. To refine the galaxy distance value, a much higher resolution, which is achievable with the Hubble space telescope, is needed.

UGC $8651=$ DDO 181. The galaxy has a curved bow-like shape very similar to DDO 165 (Karachentsev et al. 1991). Some crowded, semi-resolved stellar complexes are seen in its body. The three brightest blue stars have the mean apparent magnitude $\langle B(3 B)\rangle=20.46$ which yields a distance $D=3.4 \mathrm{Mpc}$. If the brightest one (No. 12) is not a single star but a multiple one, the galaxy distance increases up to $5.4 \mathrm{Mpc}$.

UGC $8760=$ DDO 183 . The object has an overall symmetric shape. It was resolved into stars by Hopp \& Shulte-Ladbeck (1995). The distance modulus, which is derived from the brightest blue stars (28.54) agrees well with the red one $\left(28^{\mathrm{m}} 49\right)$. 
UGC 8833. The galaxy core looks much more irregular than its outer parts. Our photometry reveals a slight increase in the colour (from +0.25 to +0.32 ) along the galaxy radius, which may be caused by the presence of different stellar generations. In the central part of the system, we distinguish about two dozen blue stars brighter than $V=24$ mag. The three brightest ones yield a galaxy distance $D=3.2 \mathrm{Mpc}$.

\section{Conclusions}

Using the large size CCD images taken in $B$ and $V$ bands with a seeing of one arcsec, we have done stellar photometry of 18 dwarf galaxies situated in the Canes Venatici cloud direction. Most of them were resolved into stars for the first time. We determined the galaxy distances, using their brightest blue stars as a distance indicator. In spite of their low radial velocities, $V_{0}<500 \mathrm{~km} / \mathrm{s}$, some galaxies (UGC 6782, UGC 7131 and, probably, K 215), have distances around $15 \mathrm{Mpc}$. Located on the southern edge of the CVn cloud they may actually belong to the outskirts of the Virgo cluster.

For two LSB galaxies, K 200 and K 215, their distances may be overestimated, probably because of a lack of young massive stars. For the remaining objects the distance estimates range from 2.3 to $8.0 \mathrm{Mpc}$, which indicates their membership in the $\mathrm{CVn}$ cloud. The ratio of the median radial velocity, $V_{0}=+304 \mathrm{~km} / \mathrm{s}$, to the median distance, $D=4.3 \mathrm{Mpc}$, leads to a local value of the Hubble parameter, $H=71(\mathrm{~km} / \mathrm{s}) / \mathrm{Mpc}$. This is larger than the cosmological value, because of the local peculiar velocity field. As the CVn cloud is only about 25 degrees from the direction of Virgo, a rough correction may be obtained from Eq. (5) in Teerikorpi et al. (1992), with $\alpha=2, r_{\text {Vir }}=$ $20 \mathrm{Mpc}, L_{\mathrm{LG}}=200 \mathrm{~km} / \mathrm{s}$. This gives $H_{0}=61 \mathrm{~km} / \mathrm{s} / \mathrm{Mpc}$.

All the considered CVn cloud members are dwarf irregular galaxies with a typical colour $(B-V)_{\mathrm{T}}=+0.32$. Some of them (UGC 7605, UGC 7639, UGC 8638, and UGC 8833) show a systematic increase of the colour outwards from their centre. Like other nearby galaxies: NGC 1705, NGC 2915 (Mackie \& Meurer 1992), and UGC 1104 (Karachentsev et al. 1996), these objects belong, probably, to a particular kind of dwarfs, which have an underlying low surface brightness old disk with a superimposed central star-forming region.

Among the studied objects, there is an intrinsically faint $\left(M_{\mathrm{T}}=-11.4\right)$ and low surface brigthess galaxy, UGCA 292, with an unusually blue integrated colour, $(B-V)_{\mathrm{T}}=+0.08$. According to the HI data by Lo et al. (1993), it has also an unusually high HI mass-toluminosity ratio, $6 M_{\odot} / L_{\odot}$. Because the fraction of its $\mathrm{HI}$ mass accounts for about $70 \%$ of the total mass, this very blue nearby dwarf galaxy may be considered as one of the youngest known objects in the Local Universe.
We have to note finally that some of the galaxies (UGC 8638, UGC 8651 and UGC 8833) should be observed again with a higher resolution to improve their distance estimates.

Acknowledgements. The authors thank S.S. Kajsin for his participation in the data processing. This work is partially supported by a INTAS-RFBR grant No. 95-IN-RU-1390.

\section{References}

Arp H., 1966, Atlas of Peculiar Galaxies, ApJS 14, 1

Bresolin F., Capaccioli M., Piotto G., 1993, AJ 105, 1779

Carignan C., Beaulieu S., 1989, ApJ 347, 760

de Vaucouleurs G., 1975, in "Galaxies and the Universe", Sandage A., Sandage M., Kristian J. (eds.). Chicago, Univ. of Chicago Press, p. 557

de Vaucouleurs G., 1978a, ApJ 224, 14

de Vaucouleurs G., 1978b, ApJ 224, 710

Georgiev Ts.B., Karachentsev I.D., Tikhonov N.A., 1997, Lett. Sov. AJ (in press)

Hopp U., Schulte-Ladbeck R.E., 1995, A\&AS 111, 527

Karachentsev I.D., 1966, Afz 2, 81

Karachentseva V.E., 1968, Soobsch. Byurakan. Obs. 39, 62

Karachentsev I.D., Kopylov A.I., Kopylova F.G., 1994, Bull. Spec. Astrophys. Obs. 38, 15

Karachentsev I.D., Makarov D.I., 1996, AJ 111, 794

Karachentsev I.D., Tikhonov N.A., Georgiev Ts.B., Bilkina B.I., Sharina M.E., 1991, A\&AS 91, 503

Karachentsev I.D., Tikhonov N.A., 1994, A\&A 286, 718

Kraan-Korteweg R., Tammann G.A., 1979, Astron. Nachr. 300, 181

Landolt A.U., 1992, AJ 104, 340

Lo K.Y., Sargent W.L.W., Young K., 1993, AJ 106, 507

Makarova L.N., Karachentsev I.D., Georgiev Ts.B., 1997, Lett. Sov. AJ (in press)

Paturel G., Bottinelli L., Fouque P., Gouguenheim L., 1992, Catalogue of Principal Galaxies: PGC-ROM, Observatoire de Lyon

Sandage A.R., Tammann G.A., 1974a, ApJ 190, 525

Sandage A.R., Tammann G.A., 1974b, ApJ 191, 603

Sandage A.R., Tammann G.A., 1974c, ApJ 194, 223

Sandage A.R., Tammann G.A., 1974d, ApJ 194, 559

Sandage A.R., Tammann G.A., 1982, ApJ 256, 339

Shombert J.M., Bothun G.D., Schneider S.E., McGaugh S.S., 1992, AJ 103, 1107

Stetson P.B., 1987, PASP 99, 191

Teerikorpi, P., Bottinelli, L., Gouguenheim, L., Paturel, G., 1992, A\&A 260, 17

Tully R.B., 1988, Nearby Galaxies Catalogue. Cambridge, Cambridge Univ. Press

van den Bergh S., 1966, AJ 71, 922

Vennik J., 1984, Tartu Astron. Obs. Publ. 73, 1

Vorontsov-Velyaminov B.A., 1959, Atlas and Catalogue of Interacting Galaxies, Part I, Moscow University 


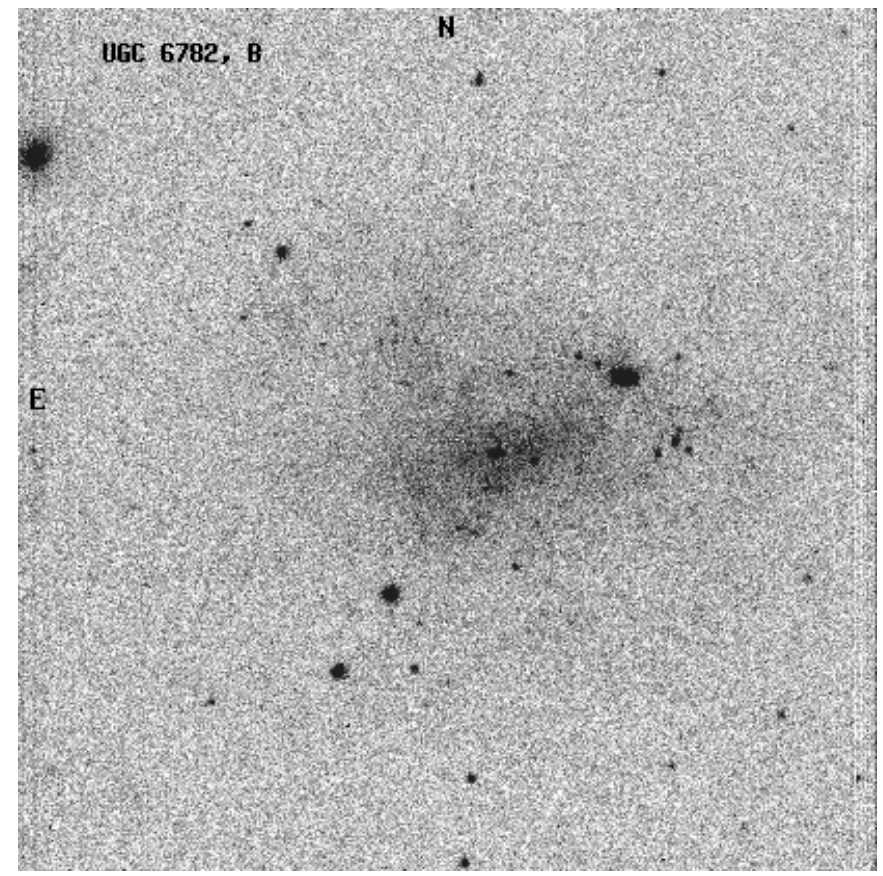

Fig. 2. The $B$ frame of UGC 6782 . As for all other images, North is top, and East is left. The upper right corner corresponds to $X=0, Y=0$ of the frame coordinates given in the tables; $X$ increases to East and $Y$ to South

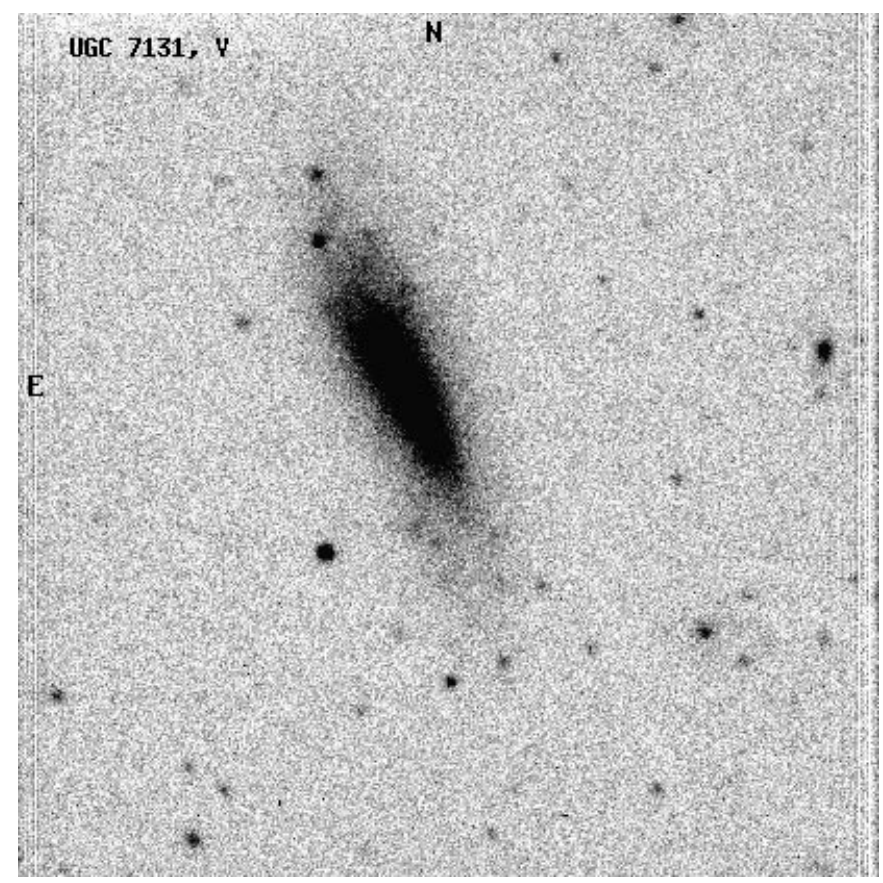

Fig. 3. The $V$ frame of UGC 7131

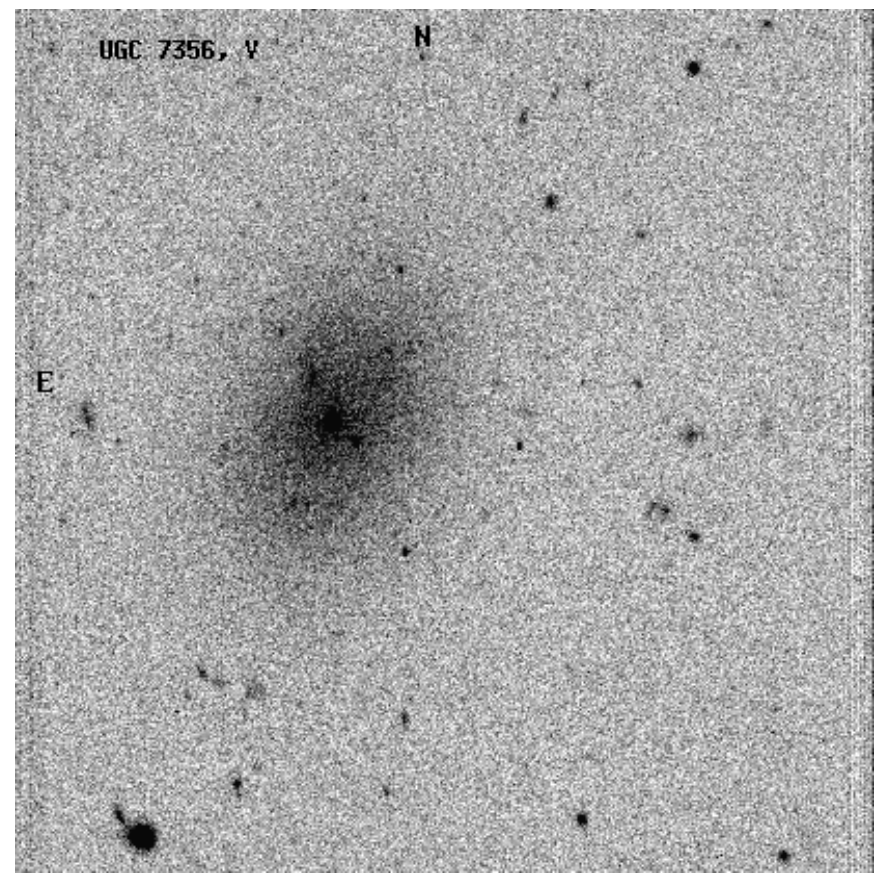

Fig. 4. The $V$ frame of UGC 7356

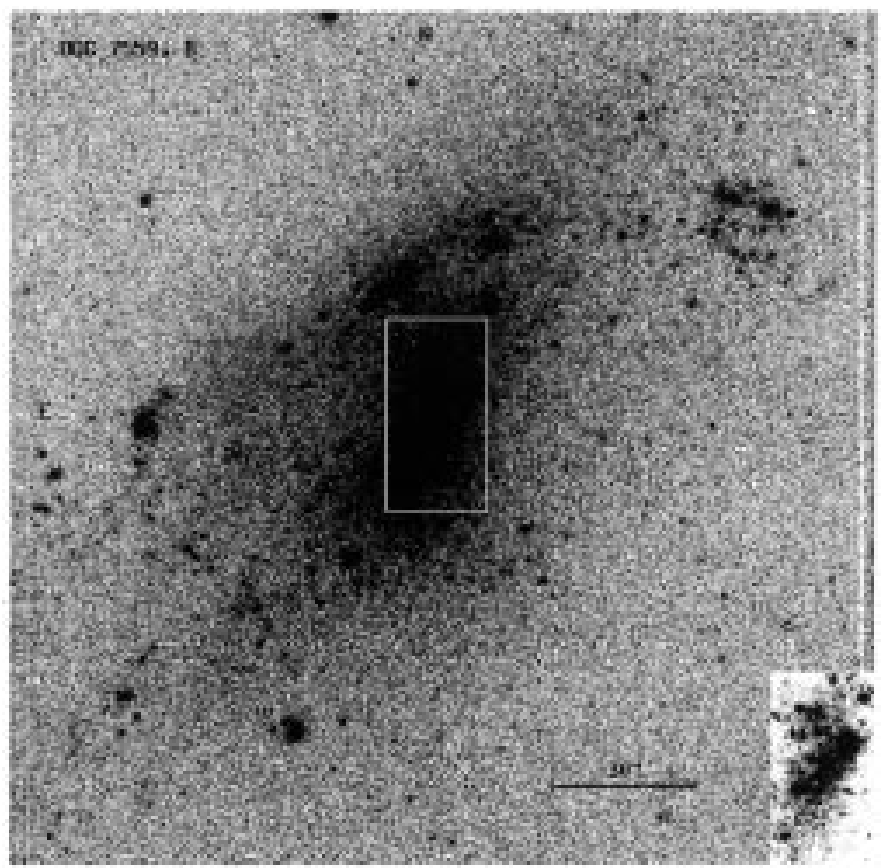

Fig. 5. The $B$ frame of UGC 7559 


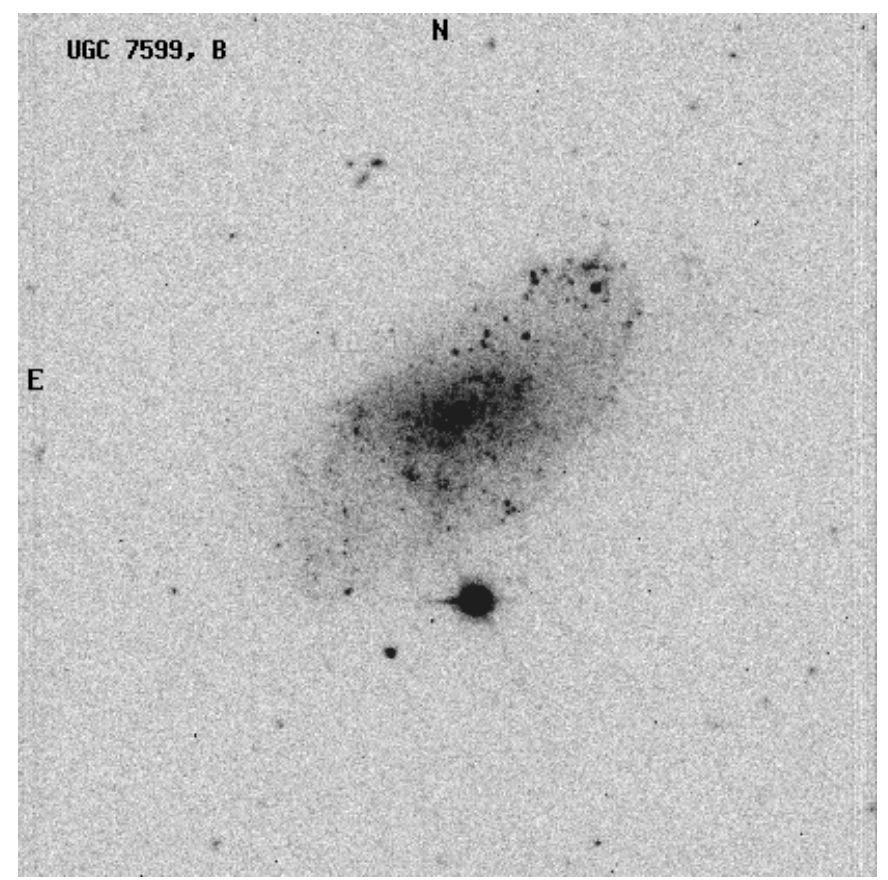

Fig. 6. The $B$ frame of UGC 7599

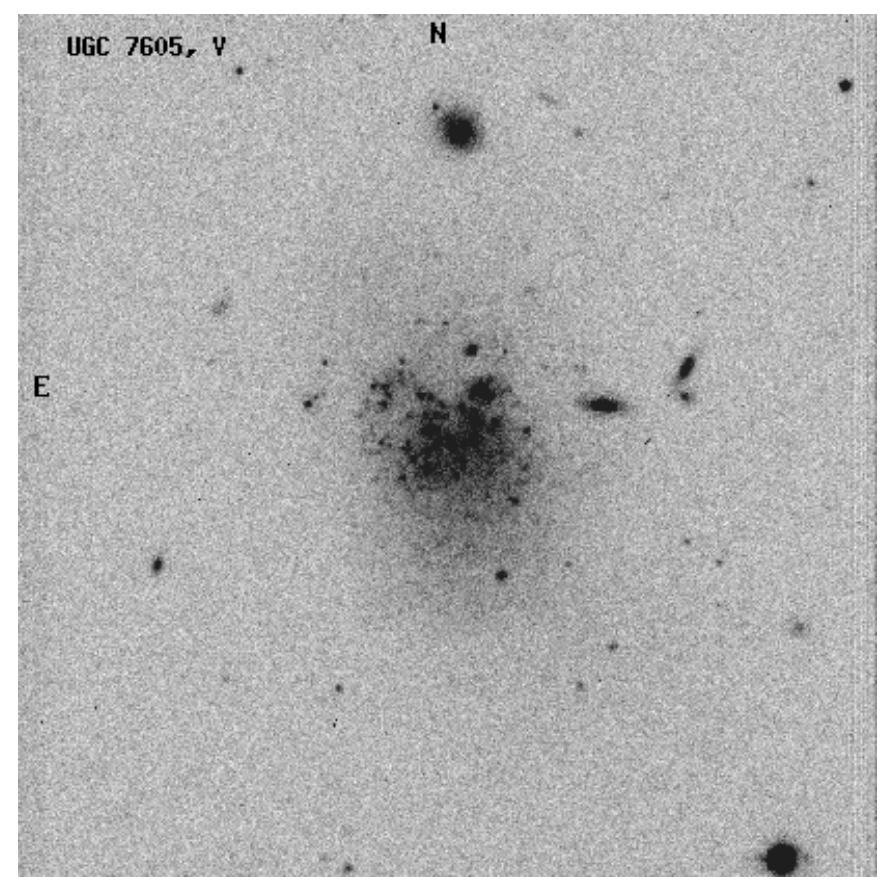

Fig. 7. The $V$ frame of UGC 7605

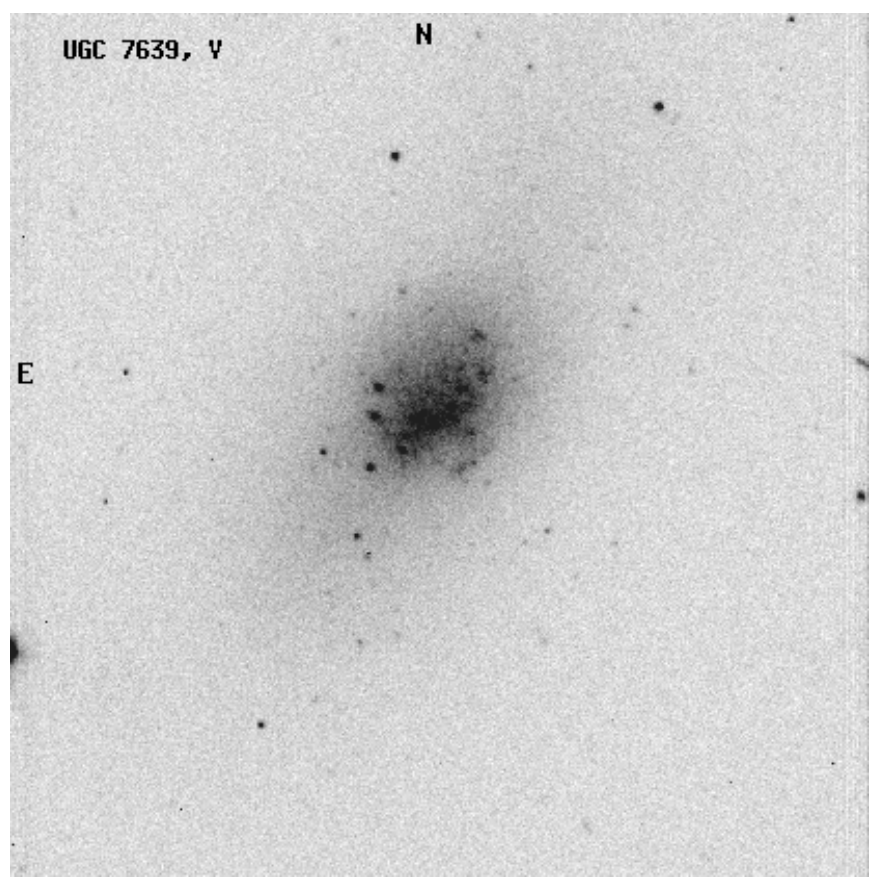

Fig. 8. The $V$ frame of UGC 7639

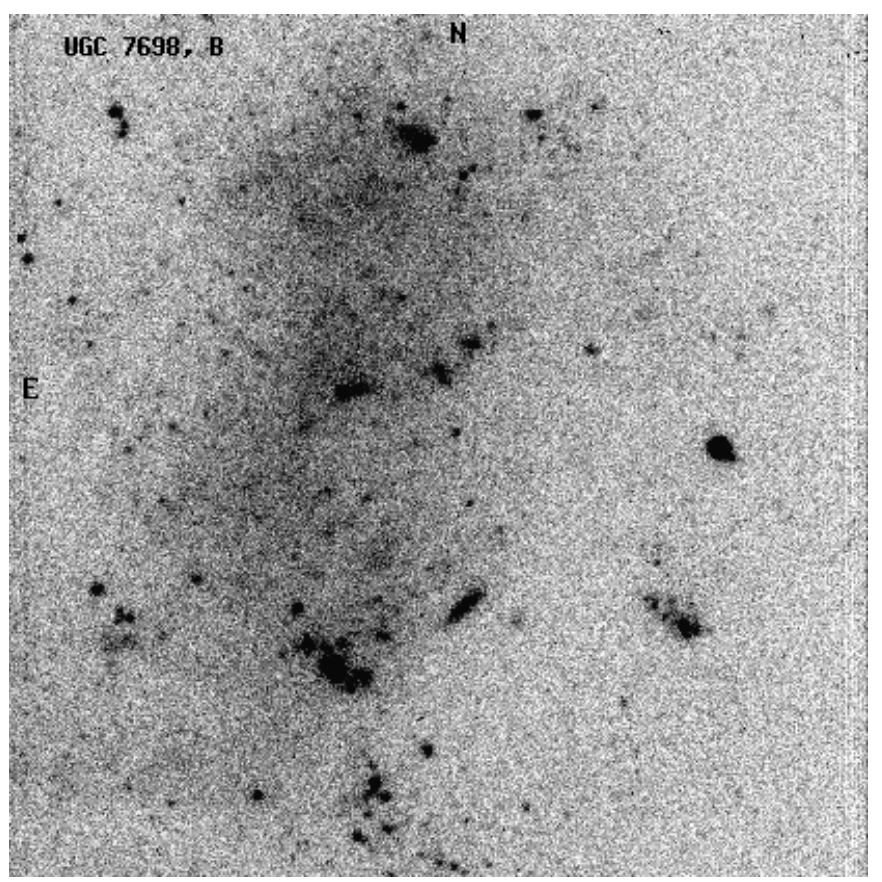

Fig. 9. The $B$ frame of UGC 7698 


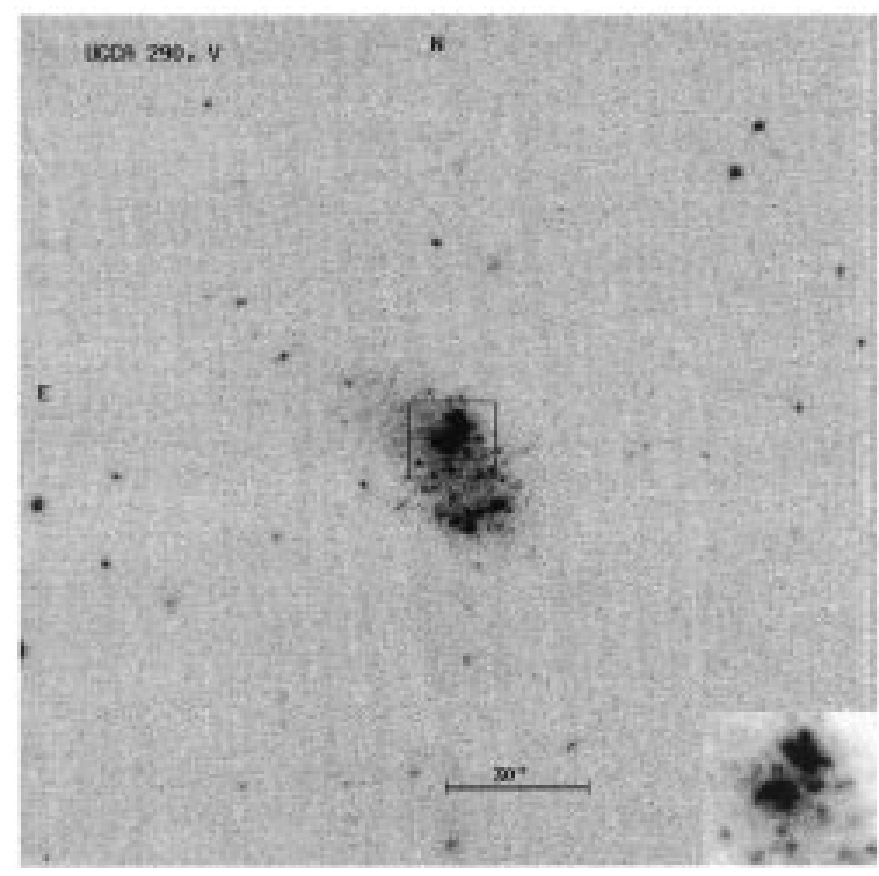

Fig. 10. The $V$ frame of UGCA 290

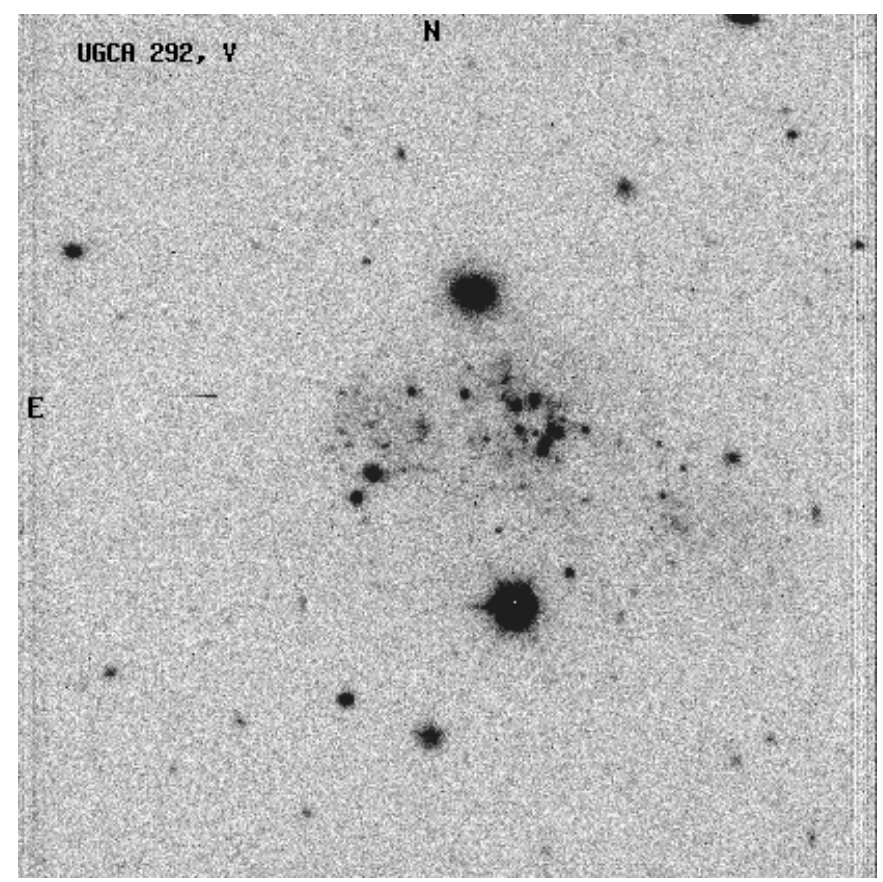

Fig. 11. The $V$ frame of UGCA 292

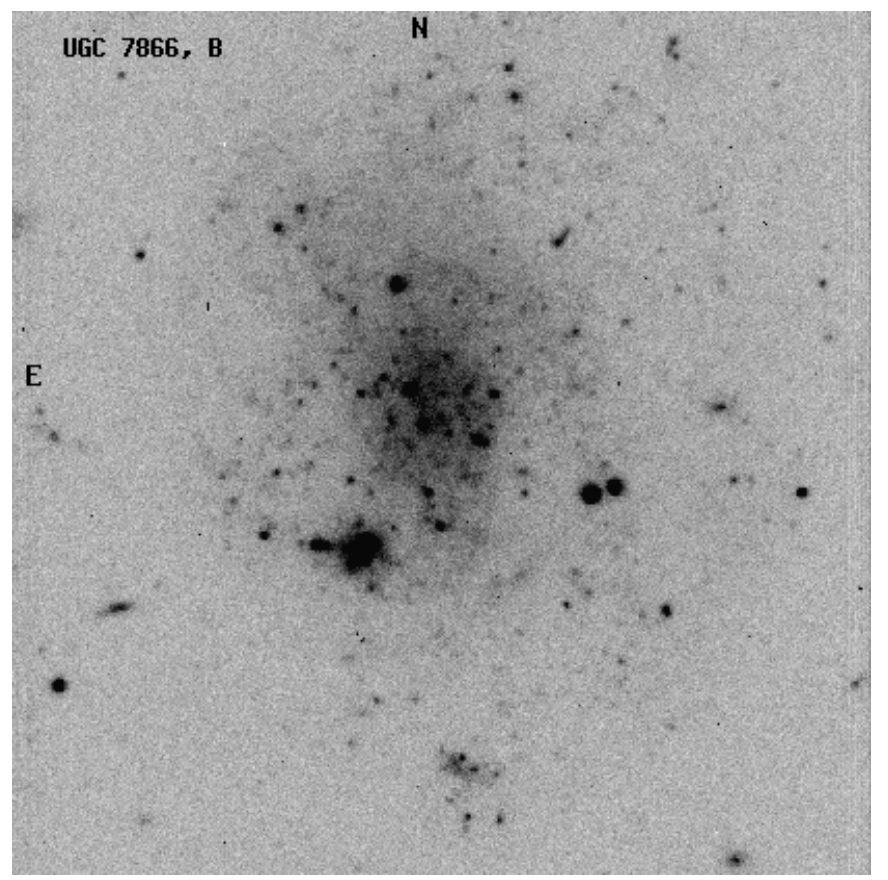

Fig. 12. The $B$ frame of UGC 7866

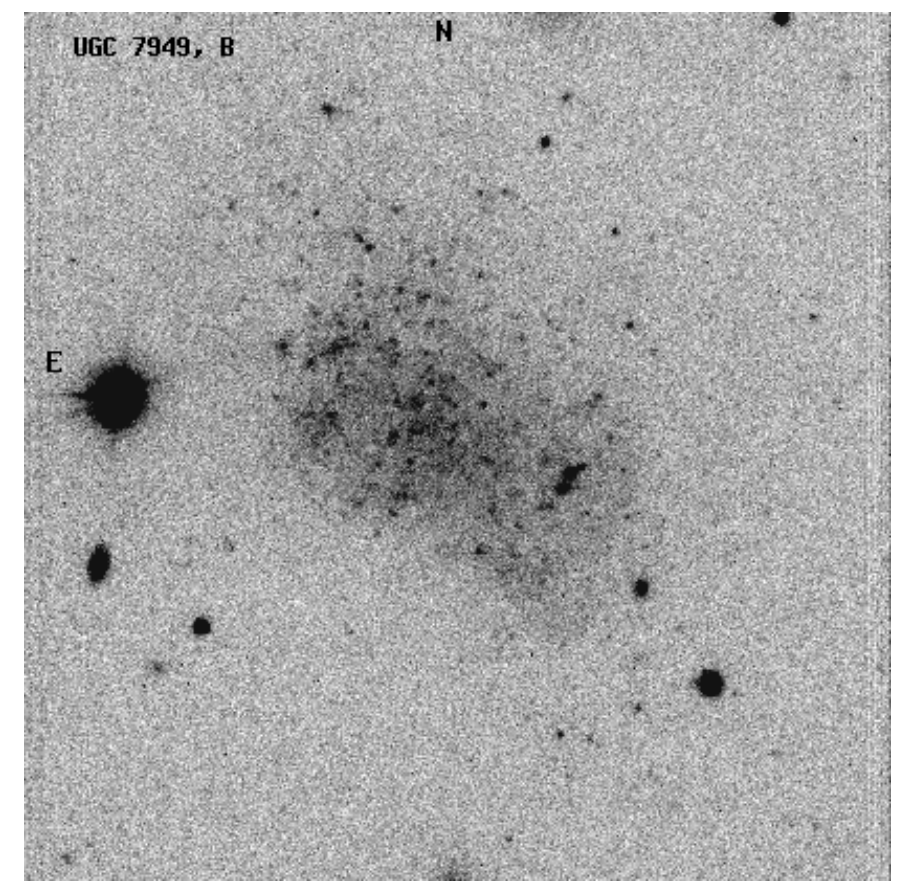

Fig. 13. The $B$ frame of UGC 7949 


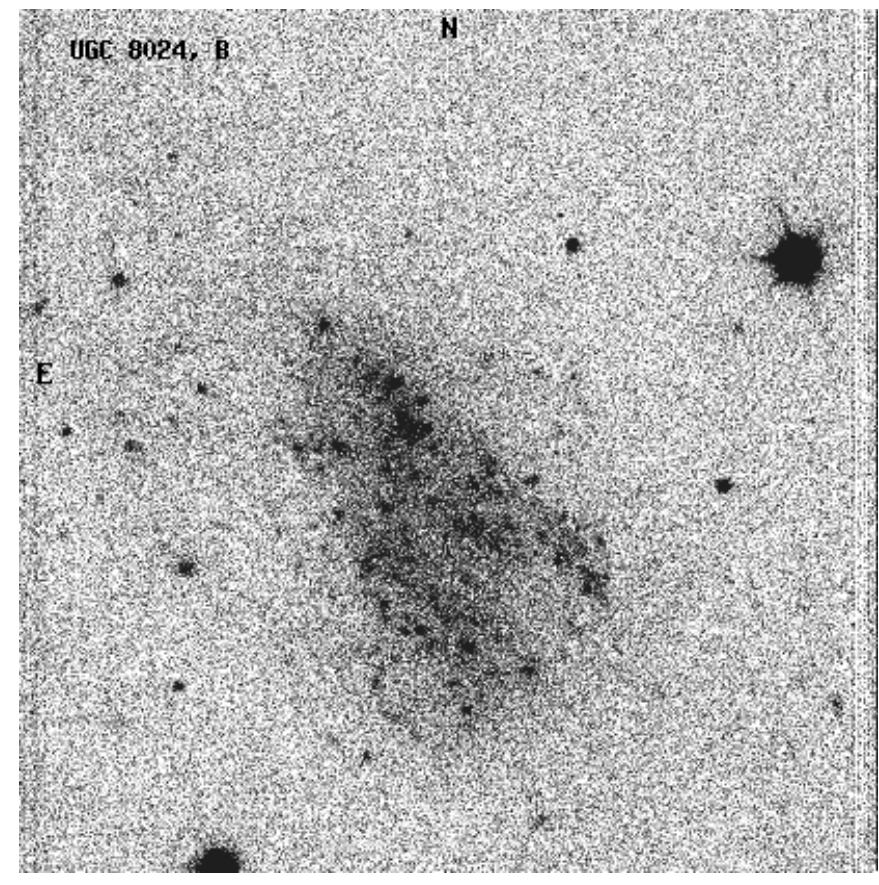

Fig. 14. The $B$ frame of UGC 8024

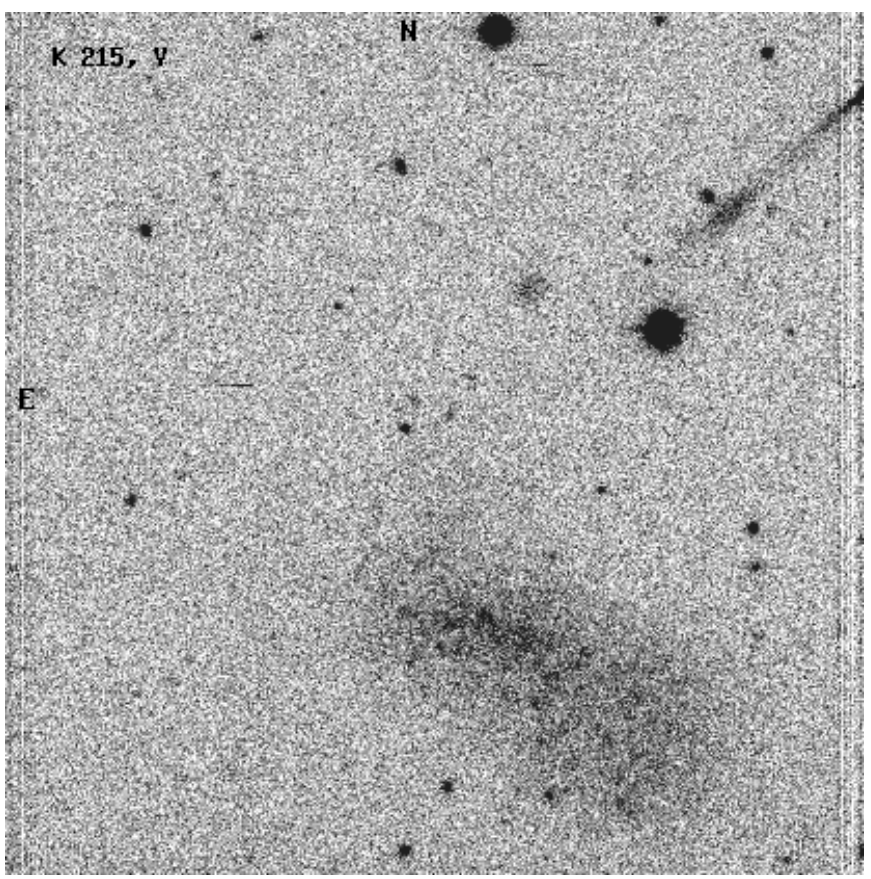

Fig. 15. The $V$ frame of $\mathrm{K} 215$

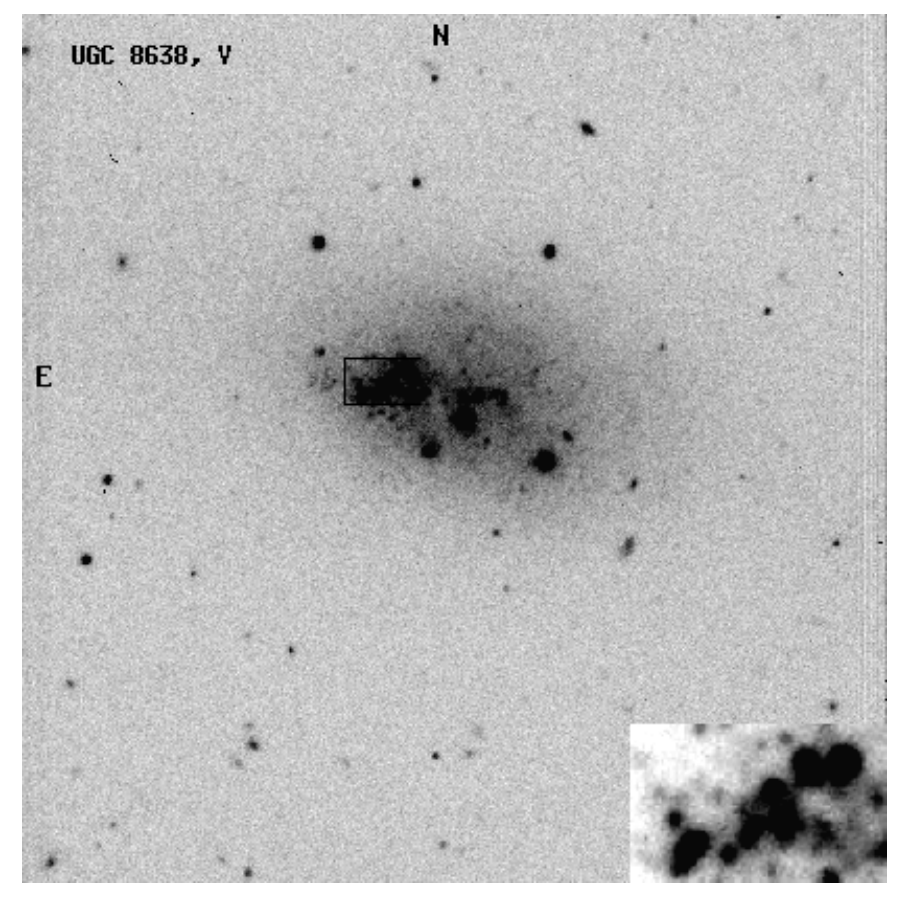

Fig. 16. The $V$ frame of UGC 8638

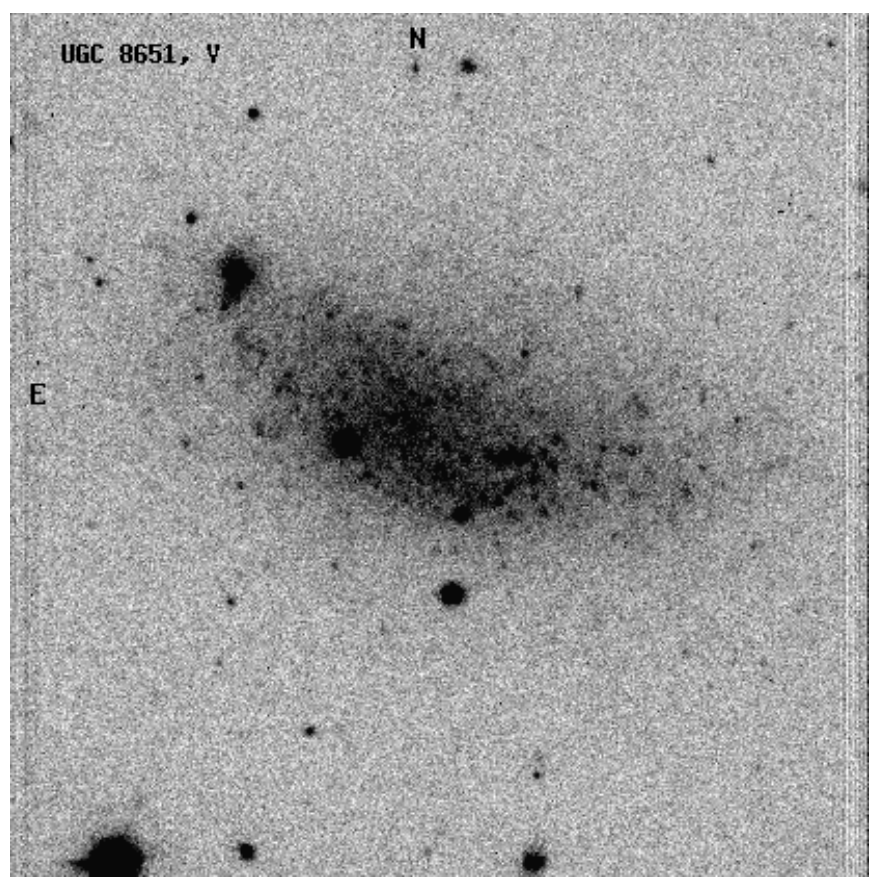

Fig. 17. The $V$ frame of UGC 8651 


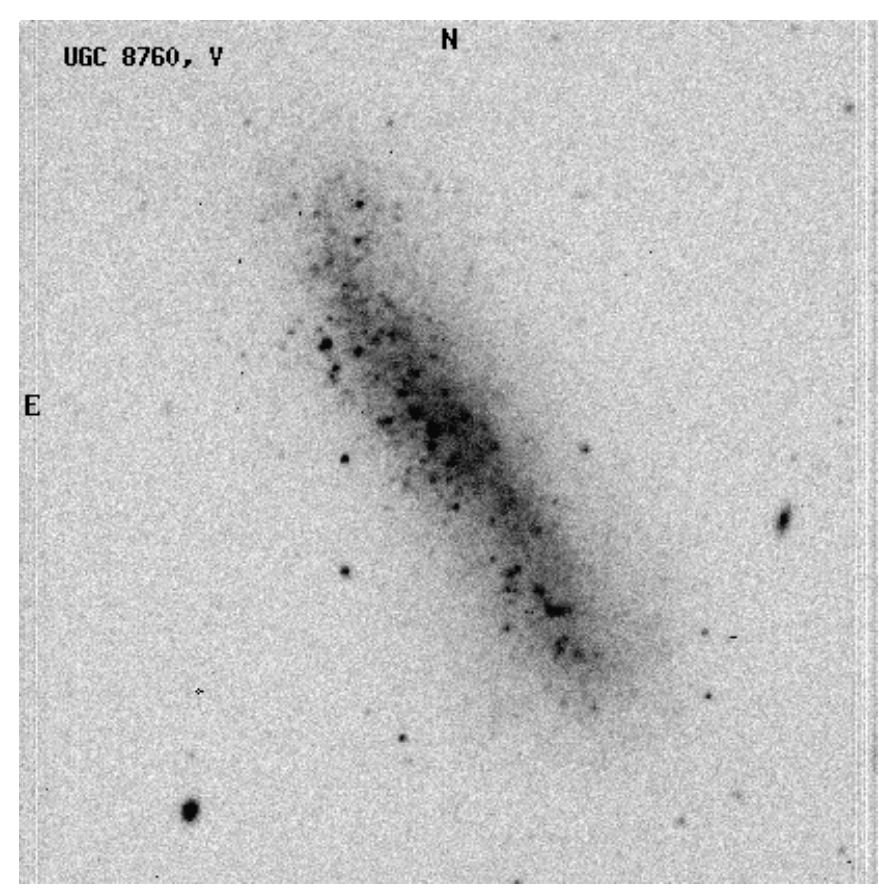

Fig. 18. The $V$ frame of UGC 8760

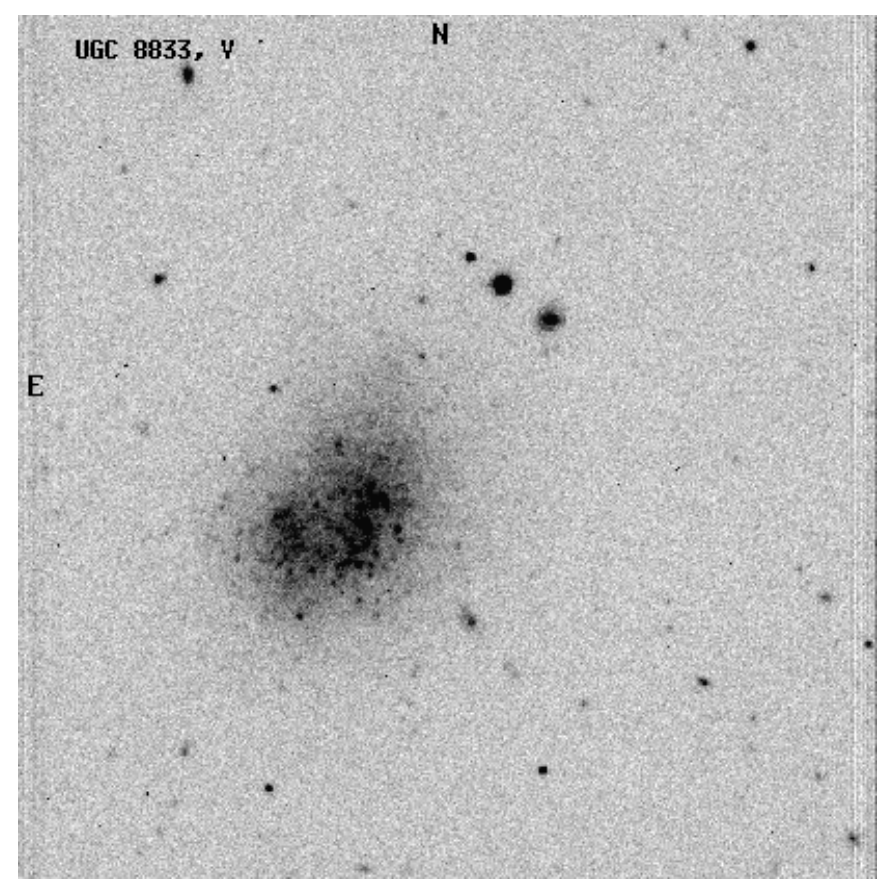

Fig. 19. The $V$ frame of UGC 8833

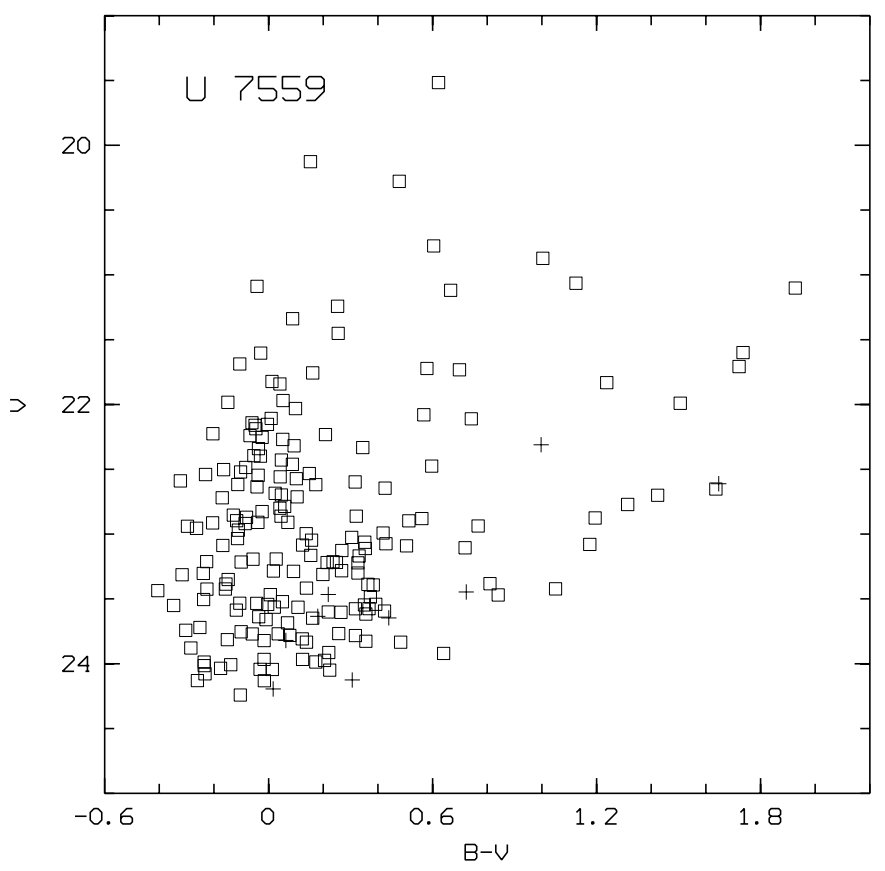

Fig. 20. $V$ vs. $(B-V)$ diagram for UGC 7559 . Here and after open squares correspond to stars measured in the main body of the galaxy and crosses indicate foreground stars

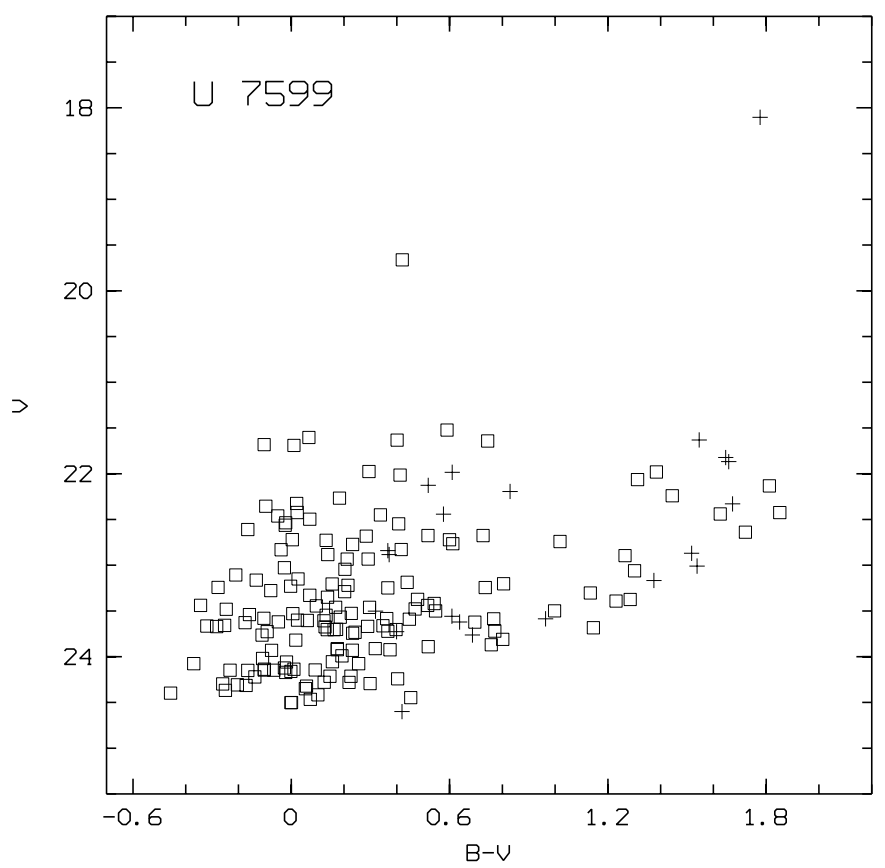

Fig. 21. $V$ vs. $(B-V)$ diagram for UGC 7599 


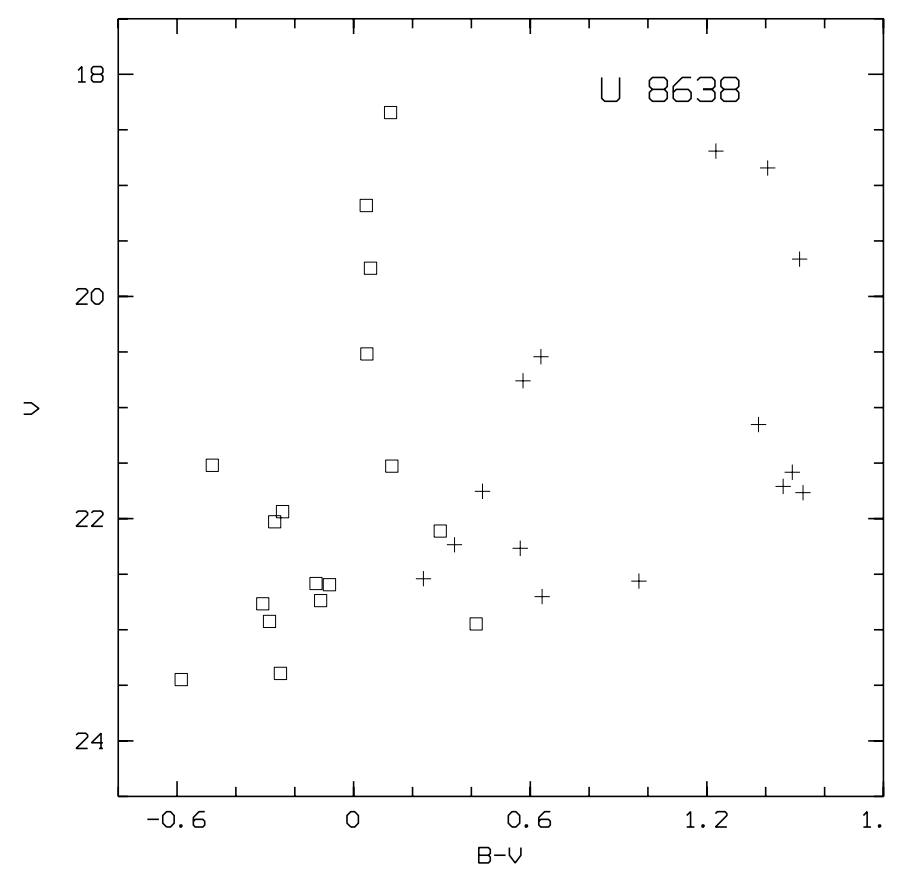

Fig. 22. $V$ vs. $(B-V)$ diagram for UGC 8638 